\title{
Association of High Expression of Mitochondrial Fission Regulator 2 with Poor Survival of Patients with Esophageal Squamous Cell Carcinoma
}

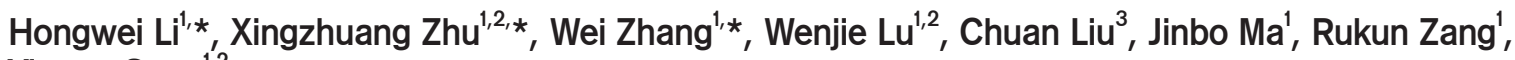 \\ Yipeng Song ${ }^{1,2}$
}

'Department of Radiation Oncology, Yantai Yuhuangding Hospital, Qingdao University, Yantai, ${ }^{2}$ Department of Oncology, School of Medicine, Qingdao University, Qingdao, ${ }^{3}$ Department of Otorhinolaryngology, The First Affiliated Hospital of Chongqing Medical University, Chongqing, China

\begin{abstract}
Mitochondrial fission regulator 2 (MTFR2) is associated with mitochondrial fission, while few studies have assessed the associations between MTFR2 expression and clinical characteristics or prognosis of esophageal squamous cell carcinoma (ESCC). In this study, we compared the expression of MTFR2 in 6 ESCC tumors and relative normal tissues by immunohistochemistry (IHC). To assess the effect of MTFR2 expression on clinicopathologic characteristics and survival, 115 paraffin embedded ESCC tissue samples were assessed by IHC staining. Furthermore, the association between clinicopathological properties and MTFR2 expression in patients with ESCC was examined. The survival analysis was performed using the Cox regression models. We found that MTFR2 expression was significantly increased in ESCC tumors compared with normal esophageal epithelial cells. IHC analysis of 115 paraffin embedded ESCC tumor specimens of the patients showed that the expression of MTFR2 was significantly associated with clinical stage $(P<0.001)$, tumor classification $(P<0.001)$, histological grade $(P<0.001)$, and other clinicopathological characteristics. Both univariate and multivariate analyses showed that MTFR2 expression was inversely correlated with the survival of ESCC patients. In conclusion, the expression of MTFR2 is significantly associated with clinicopathologic characteristics and prognosis of ESCC. Thus, MTFR2 expression could serve as a potentially important prognostic biomarker and clinical target for patients with ESCC.
\end{abstract}

Key Words MTFR2, Esophageal squamous cell carcinoma, Immunohistochemistry, Prognosis

\section{INTRODUCTION}

Esophageal cancer is one of the most common malignant tumors in the upper digestive tract of the chest $[1,2]$. Esophageal squamous cell carcinoma (ESCC) is among the most common types of upper gastrointestinal cancer [3,4]. Approximately 300,000 people die of esophageal cancer annually worldwide, with more than half in China [5]. Moreover, the mortality of esophageal cancer is ranked fourth in China [5]. Recurrence and metastasis of ESCC are still the main causes for death and treatment failure, and have crucial impact on the prognosis of ESCC $[6,7]$. Although considerable progress has been made in diagnosis and treatment, the relevant predictors for early diagnosis and prognosis remain lacking. Therefore, identification of molecular biomarkers will promote the development of new diagnostic and prognostic predictors for early diagnosis and personalized treatment of patients with ESCC.

Mitochondrial fission regulator 2 (MTFR2), also known as similarity 54 family member $A$ (FAM54A), belongs to the MTFR1/FAM54 family $[8,9]$. It has two subtypes and is produced by selective shearing. MTFR2 plays important roles in mitochondrial division and aerobic respiration [10], while few studies have assessed the effects of MTFR2 expression on clinicopathological characteristics and prognosis of ESCC. Some previous studies found that the high expression of

Received August 12, 2021, Revised September 20, 2021, Accepted September 23, 2021

Correspondence to Yipeng Song, E-mail: syp1972@sina.com, https://orcid.org/0000-0002-5030-9159

Check for updates

Rukun Zang, E-mail: zangrukun@163.com, https://orcid.org/0000-0002-1852-8220

*These authors are co-first authors and contributed equally to this work. 
MTFR2 was correlated with lymph node metastasis, age and prognosis of breast cancer $[11,12]$. Currently, some studies have shown that MTFR2 is overexpressed in glioblastoma (GBM) and lung cancer, and this was correlated with sex, age, smoking history, cancer stage, histological subtype, and TP53 mutation status of lung cancer $[9,13]$. However, the role of MTFR2 expression in ESCC remains unclear.

In this study, we analyzed the expression of MTFR2 in six paired ESCC and normal specimens and 115 paraffin embedded ESCC tumor samples by immunohistochemistry (IHC). We evaluated the associations of MTFR2 expression with clinicopathological characteristics of ESCC patients, and further explored the effect of MTFR2 expression on clinical outcome of ESCC.

\section{MATERIALS AND METHODS}

\section{Preparation of patients' tissue specimens}

Besides the 6-pairs of ESCC patients, additional 115 paraffin-embedded tumor specimens from patients, pathologically, diagnosed with ESCC between 2005 and 2010 at Yantai Yuhuangding Hospital, were included in this study. Detailed clinical information of the patients is shown in Table 1. Written informed consents were given to all patients before undertaking the study which was approved by the ethic committee of Yantai Yuhuangding Hospital (IRB No. 2017-204).

All procedures performed in studies involving human participants were in accordance with the ethical standards of the Ethics Committee of the Yantai Yuhuangding Hospital Affiliated Hospital of Qingdao University and with the 1964 Helsinki declaration and its later amendments or comparable ethical standards.

\section{Measurement of MTFR2 expression by IHC}

MTFR2 expression was measured in 115 paraffin embedded ESCC specimens and 6 pairs of ESCC and normal tissue samples by IHC, as previously described [14]. In IHC, we used rabbit monoclonal antibody targeting MTFR2 (1:200, Sigma $^{\circledR}$ Life Science). Samples without any antibody were used as the corresponding blank control [14]. In qualitative analysis, we selected 6 samples, which contained cancer and adjacent normal tissues, for comparison [15]. Two independent pathologists who were not aware of the relevant clinical information evaluated the results of $\mathrm{IHC}$ staining of tumor sections [16]. The positive staining score of cancer cells was divided into the following five grades: $0(0), 1(<10 \%), 2(11 \%$ to $50 \%), 3(51 \%$ to $75 \%)$, and $4(>75 \%)$. The staining intensity was graded as follows: 0 (negative = no staining), 1 (weak = light yellow), 2 (moderate = yellow brown), and 3 (strong = brown). The staining index (SI) was defined as the staining intensity score $x$ the proportion of positive staining tumor cells (ranging from 0 to 12). Simultaneously, we defined $\mathrm{SI} \geq 6$ as high expression and $\mathrm{SI}<6$ as low expression.

\section{Statistical analysis}

The relationships between MTFR2 expression levels and sex, age, T stage, lymphatic metastasis, distant metastasis (tumor-node-metastasis [TNM] stage) and clinical stage were analyzed using the Spearman correlation method. The effect of MTFR2 expression on clinicopathological characteristics was assessed using the $\chi^{2}$ test or the Fisher's exact test. For survival analysis, the effect of clinicopathological characteristics and MTFR2 expression on prognosis of ESCC were examined by the Kaplan-Meier analysis and the log rank test, and further evaluated by univariate and multivariate Cox regression analyses. All statistical analyses were performed using SPSS software version 20 (SPSS Inc., Chicago, IL, USA). Statistical significance was set at $P<0.05$.

\section{RESULTS}

\section{MTFR2 is highly expressed in ESCC tissues}

After IHC in 115 specimens, we found that MTFR2 was highly expressed in ESCC tissues. To validate the expression of MTFR2 in ESCC, we further analyzed the expression of

Table 1. Clinicopathological characteristics of patients and MTFR2 expression in ESCC

\begin{tabular}{|c|c|}
\hline Characteristics & No. of cases $(\%)$ \\
\hline \multicolumn{2}{|l|}{ Sex } \\
\hline Female & $6(5.22)$ \\
\hline Male & $109(94.78)$ \\
\hline \multicolumn{2}{|l|}{ Age (yr) } \\
\hline$>60$ & $56(48.70)$ \\
\hline$\leq 60$ & $59(51.30)$ \\
\hline \multicolumn{2}{|l|}{ Clinical stage } \\
\hline $\mathrm{I} / \mathrm{II}$ & $79(68.70)$ \\
\hline III/IV & $36(31.30)$ \\
\hline \multicolumn{2}{|l|}{ T stage } \\
\hline 1 & $20(17.39)$ \\
\hline 2 & $36(31.30)$ \\
\hline 3 & $40(34.78)$ \\
\hline 4 & $19(16.52)$ \\
\hline \multicolumn{2}{|l|}{$\mathrm{N}$ stage } \\
\hline 0 & 76 (66.09) \\
\hline 1 & $33(28.70)$ \\
\hline 2 & $6(5.22)$ \\
\hline \multicolumn{2}{|l|}{ M stage } \\
\hline 0 & $110(95.65)$ \\
\hline 1 & $5(4.35)$ \\
\hline \multicolumn{2}{|l|}{ Vital status } \\
\hline Alive & $53(46.09)$ \\
\hline Dead & $62(53.91)$ \\
\hline \multicolumn{2}{|c|}{ Histological differentiation } \\
\hline High & $22(19.13)$ \\
\hline Moderate & 67 (58.26) \\
\hline Low & $26(22.61)$ \\
\hline \multicolumn{2}{|l|}{ Expression of MTFR2 } \\
\hline Low & $77(67.00)$ \\
\hline High & $38(33.00)$ \\
\hline
\end{tabular}


MTFR2 in 6 pairs of ESCC tumor and adjacent normal tissues. Compared to that in normal tissues, the expression of MTFR2 in ESCC tissues was significantly upregulated as shown in Figure 1. In addition, to evaluate the association between MTFR2 expression and clinicopathological characteristics of patients with ESCC, we analyzed such associations in remaining 115 ESCC patients. These included 25 patients with stage I, 53 patients with stage II, 32 patients with stage III, and 5 patients with stage IV. The IHC staining showed that
38 cases $(33.00 \%)$ had high MTFR2 protein expression and 77 cases $(67.00 \%)$ had low or no expression (Table 1$)$.

\section{Association between MTFR2 expression and clinicopathologic characteristics of ESCC}

As shown in Table 2, there was no significant difference in MTFR2 expression for age and sex $(P>0.05)$, while the significant differences in MTFR2 expression were found for clinical stage $(P<0.001)$, pathological grade $(P=0.029)$, T/

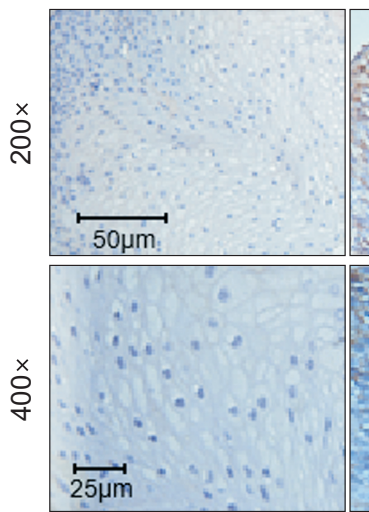

ANT

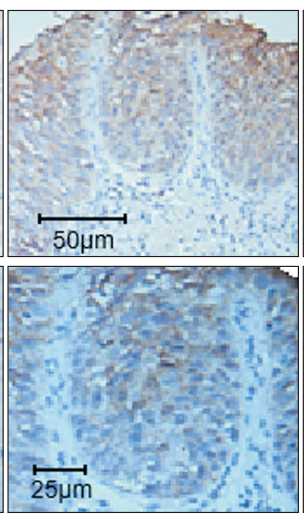

$\mathrm{T}$
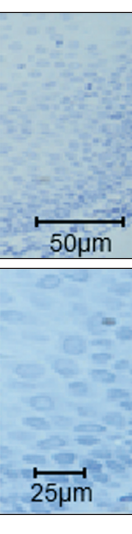

Patient 1

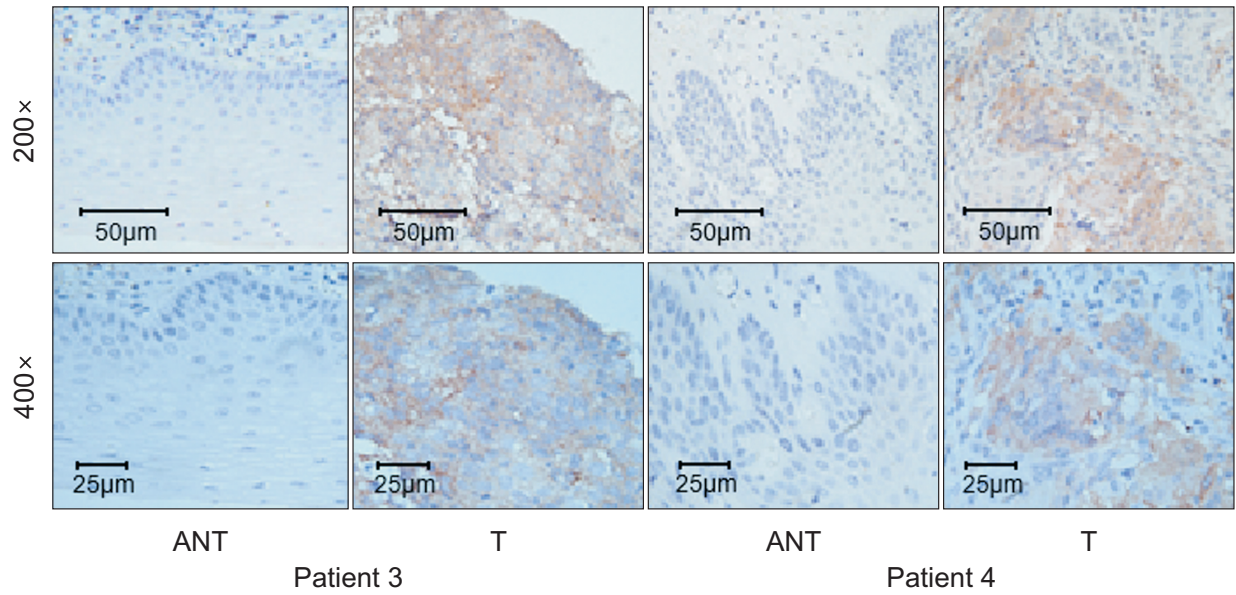

Figure 1. Upregulation of MTFR2 in ESCC tumor tissues. 
Table 2. The association of MTFR2 expression with clinicopathological characteristics of ESCC patients

\begin{tabular}{|c|c|c|c|c|c|}
\hline \multirow[b]{2}{*}{ Characteristics } & \multirow[b]{2}{*}{ No. of cases } & \multicolumn{2}{|c|}{ MTFR2 expression } & \multirow[b]{2}{*}{$\chi^{2}$ test $P$} & \multirow{2}{*}{$\begin{array}{c}\text { Fisher's exact } \\
\text { test } P\end{array}$} \\
\hline & & Low (\%) & High (\%) & & \\
\hline Sex & & & & 0.351 & 0.267 \\
\hline Female & 6 & $5(83.33)$ & $1(16.77)$ & & \\
\hline Male & 109 & $72(66.06)$ & $37(33.94)$ & & \\
\hline Age (yr) & & & & 0.213 & 0.097 \\
\hline$\leq 60$ & 56 & $40(71.43)$ & $16(28.57)$ & & \\
\hline$>60$ & 59 & $37(62.71)$ & $22(37.29)$ & & \\
\hline Clinical stage & & & & $<0.001$ & $<0.001$ \\
\hline $\mathrm{I} / \mathrm{II}$ & 79 & 65 (82.29) & $14(17.71)$ & & \\
\hline III/IV & 36 & $12(33.33)$ & $24(66.67)$ & & \\
\hline T stage & & & & $<0.001$ & $<0.001$ \\
\hline 1 & 20 & $17(85.00)$ & $3(15.00)$ & & \\
\hline 2 & 36 & $27(75.00)$ & $9(25.00)$ & & \\
\hline 3 & 40 & $27(67.50)$ & $13(32.50)$ & & \\
\hline 4 & 19 & $6(31.58)$ & $13(68.42)$ & & \\
\hline $\mathrm{N}$ stage & & & & $<0.001$ & $<0.001$ \\
\hline 0 & 76 & $60(78.95)$ & $16(21.05)$ & & \\
\hline 1 & 33 & $16(48.48)$ & $17(51.52)$ & & \\
\hline 2 & 6 & $1(16.67)$ & $5(83.33)$ & & \\
\hline M stage & & & & 0.040 & 0.037 \\
\hline 0 & 110 & $76(69.09)$ & $34(30.91)$ & & \\
\hline 1 & 5 & $1(20.00)$ & $4(80.00)$ & & \\
\hline Histological differentiation & & & & 0.029 & 0.015 \\
\hline High & 22 & $17(77.27)$ & $5(22.73)$ & & \\
\hline Moderate & 67 & $47(70.15)$ & $20(29.85)$ & & \\
\hline Low & 26 & $13(50.00)$ & $13(50.00)$ & & \\
\hline
\end{tabular}

Table 3. Spearman correlation analysis between MTFR2 expression and clinicopathologic characteristics

\begin{tabular}{lcr}
\hline Variables & $\begin{array}{c}\text { MTFR2 expression } \\
\text { level Spearman's } \\
\text { correlation }\end{array}$ & \multicolumn{1}{c}{$P$} \\
\hline Clinical stage & 0.460 & $<0.001$ \\
T stage & 0.318 & 0.001 \\
N stage & 0.373 & $<0.001$ \\
M stage & 0.213 & 0.040 \\
Vital status & 0.427 & $<0.001$ \\
Histological & 0.192 & 0.038 \\
$\quad$ differentiation & & \\
\hline
\end{tabular}

$\mathrm{N}$ stage $(P<0.001)$ and $\mathrm{M}$ classification $(P=0.040)$ (Table 2). These results suggested that MTFR2 expression was significantly associated with advanced tumors. Additionally, we also analyzed the correlations between MTFR2 expression and clinicopathologic characteristics. As shown in Table 3, there was statistically significant correlation between MTFR2 expression and clinical stage $(r=0.460, P<0.001)$ (Fig. 2), T stage $(r=0.318, P=0.001), N$ stage $(r=0.373, P<0.001)$, $M$ stage $(r=0.213, P=0.040)$, and histological differentiation $(r=0.192, P=0.038)$. Therefore, MTFR2 was significantly related to overall clinical stage, TNM stage, and poor patho- logical differentiation of ESCC, supporting a role of MTFR2 in progression of ESCC.

\section{MTFR2 expression is inversely associated with survival of ESCC}

In the patients with low MTFR2 expression, the rate of cumulative 5-year survival was $61.0 \%(95 \% \mathrm{Cl}, 60.6 \%, 75.9 \%)$, while that was only $15.8 \%(95 \% \mathrm{Cl}, 15.2 \%, 28.1 \%)$ in patients with high MTFR2 expression.

Furthermore, we found that the survival time of ESCC patients was negatively correlated with MTFR2 expression ( $r=-0.580, P<0.001)$, and the patients with high MTFR2 expression had worse survival than those with low expression (log-rank: $P<0.001$, Fig. 3A). Our stratified analysis also showed the survival differences in several subgroups. Thus, there were significant differences in survival between MTFR2 expression in patients with early disease stage (stage I/II) (log-rank, $P<0.001$, Fig. 3B) and those with advanced disease (stage III/IV) $(P<0.001$, Fig. 3C). Similarly, there were significant survival differences between the MTFR2 expression and cumulative survival of patients with T1-2, T3-4, positive lymph nodes, negative lymph nodes, non-distant metastasis or distant metastasis groups $(P<0.001$, Fig. 4). These findings in the subgroups further indicated that patients with 


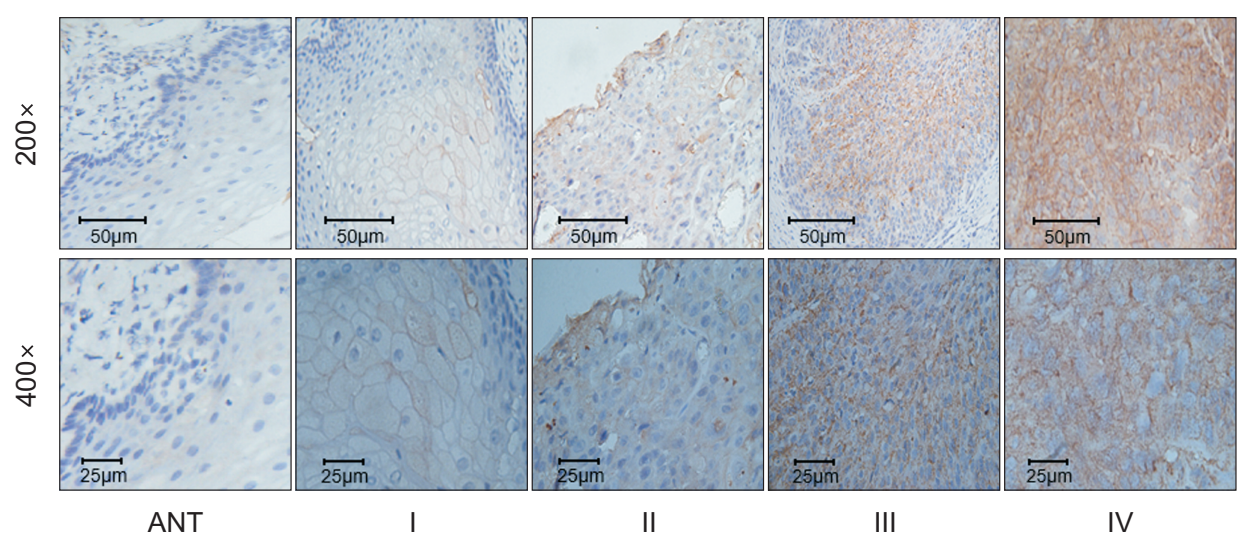

Figure 2. MTFR2 expression in normal esophagus tissues and ESCC tumor specimens at different clinical stages.
A

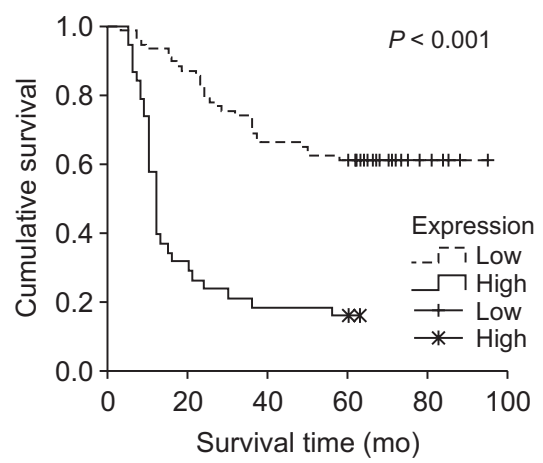

B

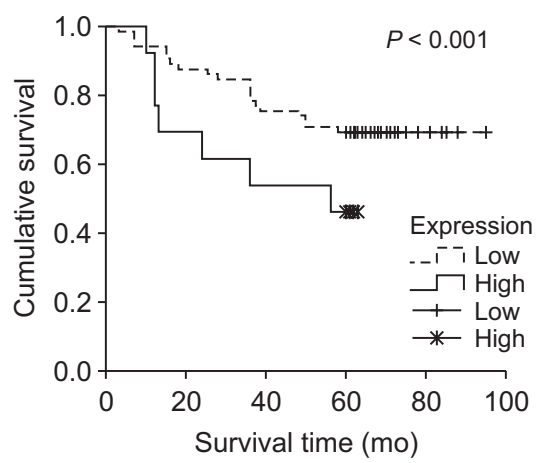

Clinical stage III-IV

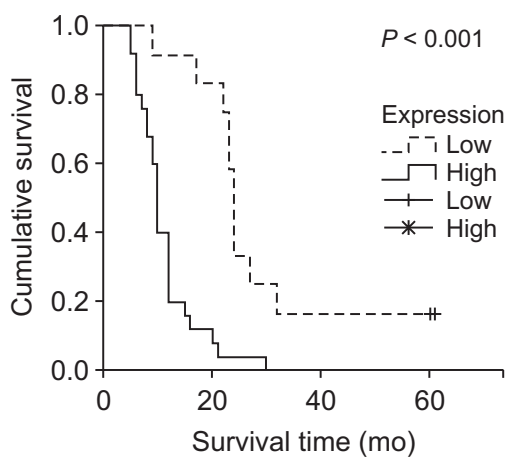

Figure 3. Effect of MTFR2 expression on survival in all patients (A), early-stage patients (B), and late-stage patients (C).

high expression of MTFR2 had shorter survival than those with low expression of MTFR2, supporting the role of MTFR2 as a potential prognostic biomarker for ESCC.

Finally, we performed both univariate and multivariate Cox regression analyses to assess the associations between MTFR2 expression and survival of patients with ESCC. Our results showed that the expression of MTFR2 was a significantly independent prognostic predictor for ESCC (adjusted hazard ratio [aHR], $3.04,95 \% \mathrm{Cl}, 1.75$ to $5.30 ; P<0.001$; Table 4).

\section{DISCUSSION}

In this study, MTFR2 expression was relatively higher in tumor lesions than that in normal tissues. MTFR2 was highly expressed in ESCC tumor specimens, and it was significantly correlated with clinical stage, T, N, M classification, histological differentiation, and metastasis of ESCC. The expression of MTFR2 increased with the progression of ESCC. Our current findings demonstrate that MTFR2 is an independent prognostic factor for the prognosis of patients with ESCC, and the patients with high MTFR2 expression have worse survival than those with corresponding low expression. Therefore, it is likely that MTFR2 expression may have a significant clinical value as a new prognostic predictor and one of potential nov- el targets for future targeted treatment of ESCC.

As a mitochondrion fission factor, MTFR2 participates in aerobic respiration and has antioxidant effects $[8,17]$. In addition, MTFR2 may play a key role in the induction of intrinsic apoptosis $[18,19]$. Compared to its role in normal cells, mitochondrion induces tumor-related programming and accelerates growth of tumor cells and blood vessels [20,21]. Some studies have shown that MTFR2 may transcriptionally regulate dual specificity protein kinase TTK [9]. MTFR2 is one of the key genes related to TTK, which can be regulated by activating the promoter of TTK $[22,23]$. Furthermore, it is involved in regulation of proliferation of glioma stem cells (GSCs) and thus may affect the prognosis of GBM $[9,20]$. Most recently, MTFR2 has been reported to promote the proliferation, migration, and invasion of oral squamous carcinoma [24], and MTFR2-dependent regulation of TTK was involved in maintaining GSCs in GBM and might serve as a potential novel druggable target for GBM. In addition to cell proliferation, TTK was also involved in centrosome duplication, DNA damage response, and organ development [25]. Another study showed that reduced TTK levels could induce abnormal mitoses, activate apoptosis and reduce survival of breast cancer [26].

TTK depletion might seriously impair the viability and clonogenic capability of triple-negative breast cancer cell lines 

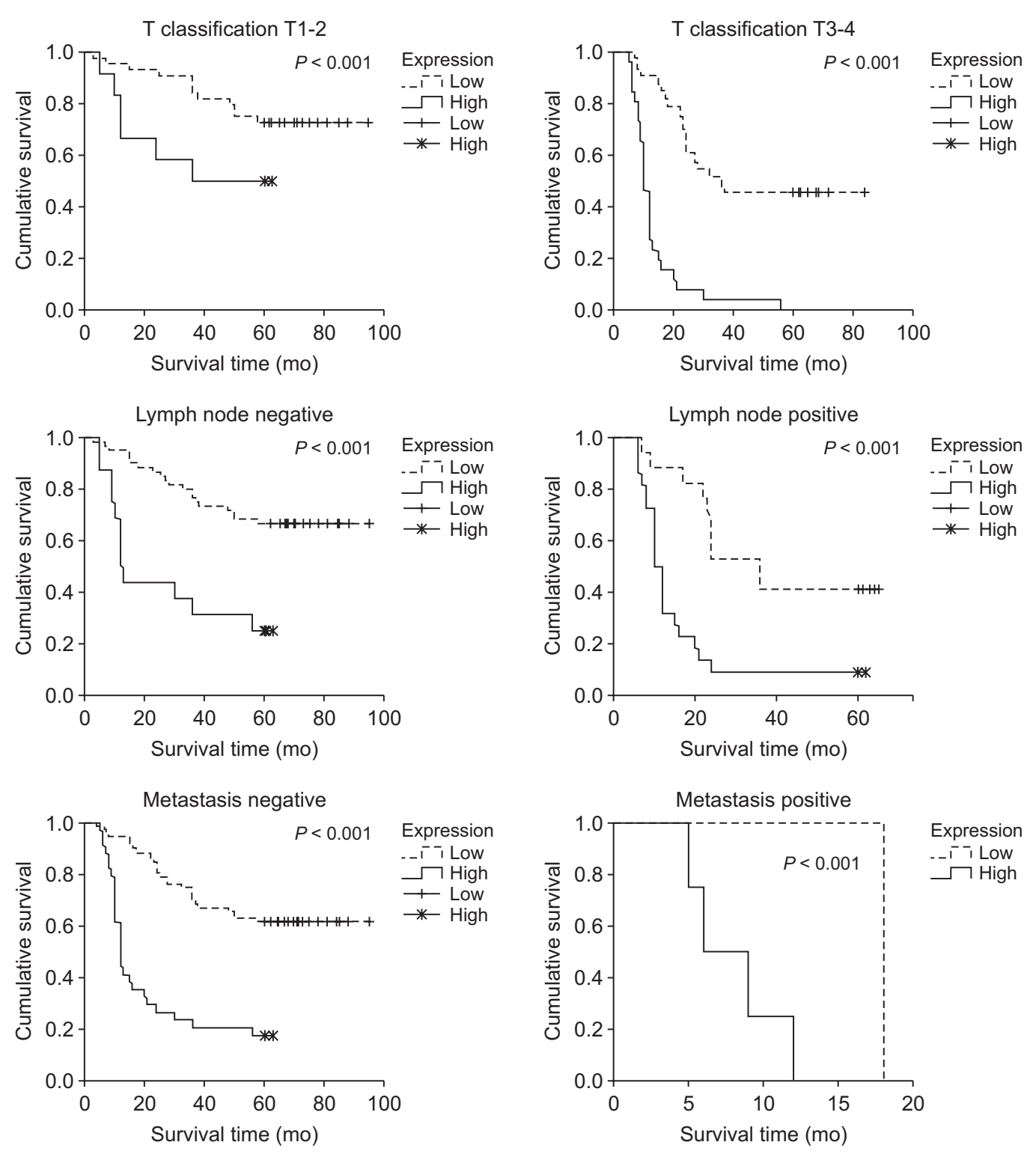

Figure 4. Effect of MTFR2 expression on survival of ESCC patients stratified by T classification, lymph node status, and metastasis status.

[27]. Thus, TTK might be an independent predictor of prognosis, and it appears biologically plausible that MTFR2 might play important roles in regulation of TTK in ESCC as well as occurrence and development of ESCC. However, the exact mechanisms underlying such associations require further exploration.

Dastsooz et al. [10] showed that the expression of MTFR2 was related to poor prognosis, suggesting that MTFR2 might be involved in the progression and invasion of several cancers [28,29]. In recent studies [11,12], MTFR2 was highly expressed in breast cancer tissue. Its altered expression affected the proliferation and metastasis of breast cancer and inhibited epithelial mesenchymal transition. Other similar studies found that the high expression of MTFR2 was related to the clinicopathology, diagnosis and prognosis, and promot- ed the occurrence and cancer development through p53-dependent cell cycle, DNA replication, and homologous recombination in lung cancer [13]. Such findings may highlight the potential role of MTFR2 in ESCC as a novel therapeutic target for treatment.

Although we found that MTFR2 was overexpressed in ESCC, and was significantly associated with advanced disease and prognosis, which was consistent with the research on glioma [9], our research has some limitations. First, we only analyzed the expression levels of cell protein, while we did not specifically analyze other aspects of genetic alterations. Second, our sample size is relatively small, and all study patients are from a single hospital. Moreover, the selection bias may also exist. Nevertheless, the current preliminary findings from this pilot study might serve as a basis for future 
Table 4. Univariate and multivariable analysis on association between MTFR2 expression and survival in patients with ESCC

\begin{tabular}{|c|c|c|c|c|c|c|c|}
\hline \multirow{2}{*}{ Variables } & \multirow{2}{*}{ No. of patients } & \multicolumn{3}{|c|}{ Univariate analysis } & \multicolumn{3}{|c|}{ Multivariate analysis } \\
\hline & & $P$ & $\mathrm{cHR}^{\dagger}$ & $95 \% \mathrm{Cl}$ & $P$ & $\mathrm{aHR}^{*}$ & $95 \% \mathrm{Cl}$ \\
\hline MTFR2 expression & & $<0.001$ & & & $<0.001$ & & \\
\hline Low & 77 & & 1.00 & & & 1.00 & \\
\hline High & 38 & & 3.12 & $1.66-5.27$ & & 3.04 & $1.75-5.30$ \\
\hline
\end{tabular}

${ }^{\dagger} \mathrm{CHR}$, crude hazard ratio; *aHR, adjusted hazard ratio; HR was adjusted by age, sex, clinical stage, T/N/M stage, and histological differentiation.

hypothesis generation and testing as well as validation in large well-designed prospective studies. Finally, more studies are needed to explore the exact molecular mechanisms underlying such associations.

In conclusion, in the current study, we found that MTFR2 is overexpressed in ESCC and correlated with ESCC progression. MTFR2 overexpression was inversely associated with survival of patients with ESCC, particularly in aggressive tumors. Thus, our current findings might support that MTFR2 could be an independent biomarker for prognosis and exploited as a potential new therapeutic target for ESCC.

\section{ACKNOWLEDGMENTS}

This article is one of the results of the Key Research and Development Plan of Yantai City (2018SFGY110).

\section{CONFLICTS OF INTEREST}

No potential conflicts of interest were disclosed.

\section{ORCID}

Hongwei Li, https://orcid.org/0000-0003-2801-4482

Xingzhuang Zhu, https://orcid.org/0000-0002-6706-2666

Wei Zhang, https://orcid.org/0000-0002-3936-4966

Wenjie Lu, https://orcid.org/0000-0003-1534-1740

Chuan Liu, https://orcid.org/0000-0003-0642-3154

Jinbo Ma, https://orcid.org/0000-0002-1646-9466

Rukun Zang, https://orcid.org/0000-0002-1852-8220

Yipeng Song, https://orcid.org/0000-0002-5030-9159

\section{REFERENCES}

1. Torre LA, Bray F, Siegel RL, Ferlay J, Lortet-Tieulent J, Jemal A. Global cancer statistics, 2012. CA Cancer J Clin 2015;65:87-108.

2. Zhong L. Research progress on radiotherapy of esophageal carcinoma. Chin J Clin Oncol 2016;43:527-30.

3. Ren QG, Yang SL, Hu JL, Li PD, Chen YS, Wang QS. Evaluation of HO-1 expression, cellular ROS production, cellular proliferation and cellular apoptosis in human esophageal squamous cell carcinoma tumors and cell lines. Oncol Rep 2016;35:2270-6.

4. Hsieh MS, Yang PW, Wong LF, Lee JM. The AXL receptor tyrosine kinase is associated with adverse prognosis and distant metastasis in esophageal squamous cell carcinoma. Oncotarget 2016;7:36956-70.

5. Lin Y, Totsuka Y, He Y, Kikuchi S, Qiao Y, Ueda J, et al. Epidemiology of esophageal cancer in Japan and China. J Epidemiol 2013;23:233-42.

6. Kleinberg L, Forastiere AA. Chemoradiation in the management of esophageal cancer. J Clin Oncol 2007;25:4110-7.

7. Han X, Lu N, Pan Y, Xu J. Nimotuzumab combined with chemotherapy is a promising treatment for locally advanced and metastatic esophageal cancer. Med Sci Monit 2017;23:412-8.

8. Monticone M, Panfoli I, Ravera S, Puglisi R, Jiang MM, Morello R, et al. The nuclear genes Mtfr1 and Dufd1 regulate mitochondrial dynamic and cellular respiration. J Cell Physiol 2010;225:767-76.

9. Wang J, Xie Y, Bai X, Wang N, Yu H, Deng Z, et al. Targeting dual specificity protein kinase TTK attenuates tumorigenesis of glioblastoma. Oncotarget 2017;9:3081-8.

10. Dastsooz H, Cereda M, Donna D, Oliviero S. A comprehensive bioinformatics analysis of UBE2C in cancers. Int $\mathrm{J}$ Mol Sci 2019;20:2228.

11. Lu G, Lai Y, Wang T, Lin W, Lu J, Ma Y, et al. Mitochondrial fission regulator 2 (MTFR2) promotes growth, migration, invasion and tumour progression in breast cancer cells. Aging (Albany NY) 2019;11:10203-19.

12. Lu W, Zang R, Du Y, Li X, Li H, Liu C, et al. Overexpression of MTFR2 predicts poor prognosis of breast cancer. Cancer Manag Res 2020;12:11095-102.

13. Chen C, Tang Y, Qu WD, Han X, Zuo JB, Cai QY, et al. Evaluation of clinical value and potential mechanism of MTFR2 in lung adenocarcinoma via bioinformatics. BMC Cancer 2021;21:619.

14. Li J, Gong LY, Song LB, Jiang LL, Liu LP, Wu J, et al. Oncoprotein Bmi-1 renders apoptotic resistance to glioma cells through activation of the IKK-nuclear factor-кB Pathway. Am J Pathol 2010;176:699-709.

15. Andl CD, Mizushima T, Nakagawa H, Oyama K, Harada $H$, Chruma K, et al. Epidermal growth factor receptor mediates increased cell proliferation, migration, and aggregation in esophageal keratinocytes in vitro and in vivo. J Biol Chem 2003;278:1824-30.

16. Yu DL, Li HW, Wang Y, Li CQ, You D, Jiang L, et al. AcylCoA dehydrogenase long chain expression is associated with esophageal squamous cell carcinoma progression and poor prognosis. Onco Targets Ther 2018;11:7643-53. 
17. Rahman J, Rahman S. Mitochondrial medicine in the omics era. Lancet 2018;391:2560-74.

18. Tonachini L, Monticone M, Puri C, Tacchetti C, Pinton P, Rizzuto $\mathrm{R}$, et al. Chondrocyte protein with a poly-proline region (CHPPR) is a novel mitochondrial protein and promotes mitochondrial fission. J Cell Physiol 2004;201:470-82.

19. Monticone M, Tonachini L, Tavella S, Degan P, Biticchi R, Palombi $F$, et al. Impaired expression of genes coding for reactive oxygen species scavenging enzymes in testes of Mtfr1/ Chppr-deficient mice. Reproduction 2007;134:483-92.

20. Jelluma N, Brenkman AB, van den Broek NJ, Cruijsen CW, van Osch MH, Lens SM, et al. Mps1 phosphorylates Borealin to control Aurora B activity and chromosome alignment. Cell 2008;132:233-46.

21. Sabharwal SS, Schumacker PT. Mitochondrial ROS in cancer: initiators, amplifiers or an Achilles' heel? Nat Rev Cancer 2014; 14:709-21.

22. Hudler P, Britovsek NK, Grazio SF, Komel R. Association between polymorphisms in segregation genes BUB1B and TTK and gastric cancer risk. Radiol Oncol 2016;50:297-307.

23. Kaistha BP, Honstein T, Müller V, Bielak S, Sauer M, Kreider R, et al. Key role of dual specificity kinase TTK in proliferation and survival of pancreatic cancer cells. Br J Cancer 2014;111:1780-7.
24. Wang W, Xiong M, Jiang L, Chen Z, Shao Y. MTFR2 promotes the proliferation, migration, and invasion of oral squamous carcinoma by switching OXPHOS to glycolysis. Front Oncol 2020;10:858.

25. Liu X, Winey M. The MPS1 family of protein kinases. Annu Rev Biochem 2012;81:561-85.

26. Daniel J, Coulter J, Woo JH, Wilsbach K, Gabrielson E. High levels of the Mps1 checkpoint protein are protective of aneuploidy in breast cancer cells. Proc Natl Acad Sci USA 2011;108:5384-9.

27. Maire V, Baldeyron C, Richardson M, Tesson B, VincentSalomon A, Gravier E, et al. TTK/hMPS1 is an attractive therapeutic target for triple-negative breast cancer. PLoS One 2013;8:e63712.

28. Nath S, Banerjee T, Sen D, Das T, Roychoudhury S. Spindle assembly checkpoint protein Cdc20 transcriptionally activates expression of ubiquitin carrier protein UbcH10. J Biol Chem 2011;286:15666-77.

29. Perrotta I, Bruno L, Maltese L, Russo E, Donato A, Donato G. Immunohistochemical analysis of the ubiquitin-conjugating enzyme UbcH10 in lung cancer: a useful tool for diagnosis and therapy. J Histochem Cytochem 2012;60:359-65. 